\title{
The asymmetry of lateral interference in visual letter identification
}

\author{
WILLIAM P. BANKS, KENNETH M. BACHRACH, and DOUGLAS W. LARSON \\ Pomona College, Claremont, California 91711
}

\begin{abstract}
Asymmetry of masking refers to the fact that a masking letter placed on the peripheral side of a target in the visual field will interfere more with recognition of the target than will a masking letter placed on the central side of the target. The experiments in this paper show that lateral masking for a pair consisting of a single target and a single mask cannot be entirely explained by processing interference caused by the mask but that masking has a component of purely sensory interference. Furthermore, the asymmetry of masking at the sensory level, when all sources of processing interference are eliminated, can be explained in terms of the falloff in acuity from the center to the periphery of the retina. The sensory component of masking is asymmetrical because, with the target at a constant retinal location, the target-plus-mask configuration is, as a whole, more peripherally located with a peripheral mask than a central one, and it is the location of the target-plus-mask configuration that determines performance.
\end{abstract}

Recognition of a single letter in the visual field can be greatly impaired by the presence in the field of other, irrelevant letters that the observer is not attempting to identify. The interference caused by irrelevant letters is quite potent in some configurations and has been a topic of study for many years. Research on the topic dates back at least to Korte (1923), and Woodworth (1938) devoted a few pages to it. The more recent literature shows a resurgence of interest in lateral interference in recognition of letters.

The parameters important in determining the degree of interference irrelevant letters will cause have been explored fairly extensively. First, the effect seems to be only partly due to short-term memory limitations or to the order of attending to elements in the field, since interference from irrelevant elements is still obtained with unlimited viewing time (Taylor \& Brown, 1972; Townsend, Taylor, \& Brown, 1971). The finding of lateral interference with unlimited viewing time also shows that strategies of reading a fading iconic trace cannot account for it. It seems that lateral interference is not entirely a product of limited central processing capacity but reflects a limitation in the resolving power of the visual system.

This research was supported by National Science Foundation Grant BMS 75-20328. We thank Bill Prinzmetal, Stan Klein, and Carol Peck for their substantive comments on the research and their editorial suggestions on the paper. We are particularly grateful to Stan Klein for making available to us the eye-movement monitoring system that allowed us to perform Experiment 3. Reprint requests should be sent to William P. Banks, Department of Psychology, Pomona College, Claremont, California 91711. K. M. Bachrach is now at the Department of Psychology, Arizona State University, Tempe, Arizona 85281.
Second, lateral masking of letters by other letters appears not to operate by simple contour interaction alone. Shaw (1969) found that a solid bar placed beside a target letter had about the same masking effect as a blank space that was placed beside the target in a control condition. A nontarget letter in the same position produced the usual lateral interference effect. While an effective masking stimulus for letter targets seems to require some of the figural characteristics of a letter not shared by a solid bar, the specific features of the mask are apparently not very important. In a lateral masking experiment, Eriksen and Eriksen (1974) varied the identity of the masking and the target letters, and the featural similarity between them, and found very little differential masking at the sensory level as a result of these variations. Their feature-specific interference effects resulted from response competition rather than sensory interference. Their experiments also showed clearly a third important fact about lateral masking, namely that lateral masking declines very rapidly as the distance between target and masking letters increases.

The fourth important phenomenon concerning lateral masking, and the topic of this study, is its asymmetry in the visual field. The asymmetry is such that a masking letter will interfere with identification of a target more if it is further from the center of the field than the target than if it is closer to the center than the target. Figure 1 illustrates the asymmetry effect. In Figure 1, a target, $T$, is shown at a constant distance to the right of a fixation point with a masking element, $M$, either closer to the fixation point or further from it than the target. The asymmetry effect is such that the target will be easier to see with the mask on the inside (i.e., on the more 


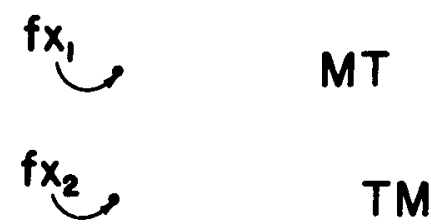

Figure 1. Target $(T)$ and mask $(M)$ configurations when target is kept at a constant distance from the fixation point (fx). The mask is central to the target for $f x_{1}$ in the figure, and it is peripheral for $\mathbf{f x}_{2}$.

foveal side) than on the outside (on the more peripheral side).

Experiments on the asymmetry effect have not used arrays like those in Figure 1. Rather, they have shown that a blank space on the peripheral side of a letter in a string facilitates identification of the letter more than does a blank space on the foveal side of the letter (Estes \& Wolford, 1971; Mackworth, 1965; Shaw, 1969; Wolford \& Hollingsworth, 1974). The procedure of using a string of letters in each array and having subjects read out as many as they can raises the possibility that memory or readout processes contribute to the effects in some way, even when counterbalancing over the experimental variables is employed. The use of long strings in the array also makes it difficult to know the extent to which the nonadjacent masking letters influence the asymmetry effect. Furthermore, in these arrays the masking letters are themselves targets and must be identified, and the asymmetry effect could arise from some sort of processing interference rather than from a strictly perceptual interaction among them. The Taylor and Brown (1972) and Townsend (1971) studies probably eliminate most effects of memory limitations because they allow unlimited viewing, but they still use large arrays, require processing of both masks and targets, and do not separate the interference caused by adjacent elements from that caused by more distant ones. They are also subject to a confounding between asymmetry of masking and retinal position that will be discussed below.

The present experiments use arrays with only two characters in order to overcome some of the interpretational problems found with large arrays of characters. To reduce the probability that interference results from subjects attempting to process the mask, the mask was always the same letter, an uppercase " $\mathrm{H}$." In Experiment 1, the pair of letters was presented briefly and the dependent variable was detection accuracy in a four-alternative forcedchoice test. In Experiment 2, the target was presented for an unlimited viewing time with a single masking letter on the foveal or peripheral side of it. While the subject viewed a fixation point, the mask was slowly moved away until the subject could see the target clearly, and the dependent variable was the threshold target-mask-distance required for clear visibility of the target. These two situations test for a purely sensory component of the asymmetry effect, because the measures of lateral interference in these experiments are unaccompanied by many of the extraneous factors that may influence performance when large arrays or whole report proceduies are used. Experiment 3 is a partial replication of Experiment 2, with monitoring of subjects' eye movements to prevent eye movements from contaminating the data.

These experiments have a second function, and that is to evaluate one interpretation of the asymmetry effect. This interpretation is based on the falloff in acuity as distance on the retina from the fovea increases. If the subject, to identify the target, must distinguish its features from those of the mask, then it is the distance of both items (or of the cluster formed by the target and mask together) from the fixation point that determines performance and not just the distance of the target. The target-mask configurations in Figure 1, by this analysis, contain an important confounding: When the mask is peripheral to the target, the array as a whole is more peripheral, and therefore in a region of lower retinal acuity, than when the mask is on the foveal side of the target. The asymmetry effect. is thus at least partly an artifact of the central-peripheral retinal acuity gradient. The procedure of using large arrays of letters, rather than a simple target-mask pair, should exacerbate the acuity difference between peripheral and foveal masks, since the long strings of noise items extend the whole array even more toward the center or periphery of the field.

Figure 2 shows arrays with targets ( $T$ ) and masks (M) constructed so as to avoid the confounding seen in Figure 1. The target-mask cluster is at the same distance from the fixation point $\left(f_{x}\right)$ when the mask is inside and outside of the target. Here a different confounding is created, however, since the target letter is further from the fixation point when the mask is on the inside than when it is on the outside. The present study uses arrays like those in Figure 1 so that the retinal position of the target will not vary with the position of the mask. The acuity gradient hypothesis of the asymmetry of interference has slightly different predictions for Experiments 1 and

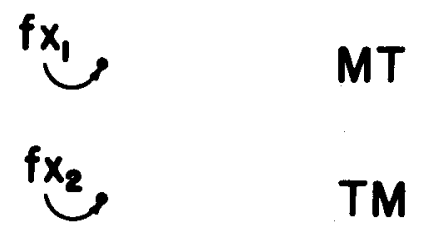

Figure 2. Target and mask configurations similar to those in Figure 1, but with the target-plus-mask configuration kept at a constant distance from the fixation point as mask is put on the peripheral or central side of the target. 
2 , and these will be discussed in the context of each experiment. The essential idea of the prediction is that the unequal masking effects of noise inside and outside the target can be eliminated, if the hypothesis is right, by correcting for the difference in mean retinal position in the two cases. The theories of Estes, Allmeyer, and Reder (1976) and Wolford (1975) also attribute at least a component of lateral masking to confusions of features in the mask and target. Both theories assume, in addition, that there is a peripheral-central gradient of masking that makes a peripheral mask more potent than a central one. If the proposed correction procedure removes the difference between mask-central and maskperipheral clusters, then the additional assumption of a peripheral-central gradient is unnecessary.

\section{EXPERIMENT 1}

\section{Method}

Procedure. On each of 1,280 trials, the subjects were required to decide whether an $\mathrm{A}, \mathrm{V}, \mathrm{X}$, or $\mathrm{Y}$ target had been presented in a brief tachistoscopic flash. Before each flash, they fixated a dot in a 21 -fL white field at $85 \mathrm{~cm}$ in an Iconix tachistoscope. The stimulus field ( $32 \mathrm{fL}$ ) always contained one of the four targets flanked by a single masking letter, $\mathrm{H}$, and subjects always received feedback as to the accuracy of their decision on each trial. At the beginning of each trial, the experimenter said "ready." The subject then pressed a switch that caused the stimulus display to be presented $1 / 2$ sec later.

The three experimental variables of interest are the retinal position of the target, the distance between the target and the masking $\mathbf{H}$, and the position of the mask on the inside (foveal side) or outside (peripheral side) of the target. The target could be at one of four positions, .5, 1.0, 1.5, and $2.0 \mathrm{deg}$, on either side of the fixation point. The mask could also be at four positions, $10^{\prime}, 15^{\prime}, 20^{\prime}$, and $25^{\prime}$, on either side of the target. The orthogonal combination of the variables (target position, left vs. right visual field, mask position, and inside vs. outside placement of mask relative to target) leads to 64 different conditions. Since each of the four targets appeared in all 64 conditions, there were 256 stimulus cards, each of which the subjects responded to five times in experimental trials, making the total of 1,280 data trials per subject. The 1,280 data trials were spread out over five separate 1-h sessions, usually given on different days. A complete set of all 256 different cards was given on each session. The letters were typed on a Smith Corona Classic 12 typewriter, with each letter measuring $10^{\prime}$ in height and $8^{\prime}$ in width.

Stimuli were presented in blocks of approximately 50 stimulus cards, and at a rate of about one every $5 \mathrm{sec}$. After each block of 50 , the subjects rested for 1 or $2 \mathrm{~min}$; three or more unscored trials were presented after each of the rests before data trials resumed. Practice consisted of two or three blocks of 50 trials that followed an initial duration-setting block, during which the stimulus duration was progressively reduced until the subject seemed to have a fairly stable score around $60 \%$ correct. The exposure duration ranged from 15 to $50 \mathrm{msec}$ across subjects, and it was not adjusted as the subject improved in accuracy over the data collection trials within a session.

Subjects. The first two authors, one unpaid volunteer, and three paid volunteers each served as subjects.' Two of the six subjects were female, the ages ranged from 18 to 32 years, and all had normal or corrected-to-normal vision.

\section{Results}

Figure 3 shows accuracy of detection as a function of the retinal position of the target, separately plotted for conditions with the mask on the inside of the target (that is, between the target and the fixation point) and on the outside (on the side of the target toward the periphery). Both retinal position and the asymmetry of masking (mask inside vs. mask outside) are reliable, with $F(3,15)=103$ for retinal position $(p<.001)$ and $F(1,5)=15.3$ for mask position $(p<.025)$. All six subjects were more accurate with the mask on the inside than on the outside $(p=1 / 64)$. The interaction between these two variables falls short of significance, $F(3,15)$ $=3.1$, indicating that the two functions in Figure 3 are statistically parallel. Not plotted in Figure 3 is the effect of the distance between target and mask (the effect of target-noise proximity). This effect was reliable, with $F(3,15)=15.8, p<.025$, but it did not interact significantly with any of the other variables. Mean accuracy for the four mask positions in order, starting with the one nearest to the target, was $72.3 \%$, $75.2 \%, 78.6 \%$, and $78.0 \%$ correct. The proximity effects, plotted separately for the four retinal positions, are quite parallel, $F(9,45)=1.39, \mathrm{p}>.20$.

This experiment eliminates a number of nonperceptual explanations of asymmetry of masking. Since it uses a forced-choice detection technique rather than a whole-report technique, it seems immune to explanations based on serial position effects in memory or in perceptual identification. The fact that there were only two elements in the field further reduces the possible contributions of memory factors

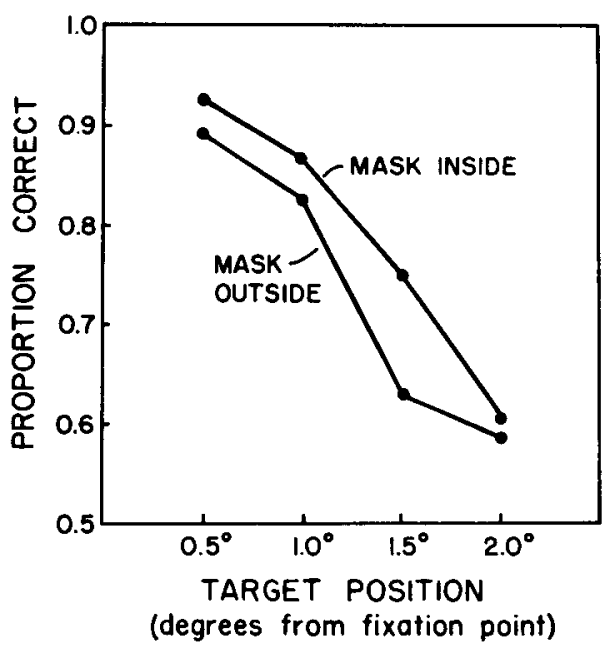

Figure 3. Detection accuracy in Experiment 1 plotted as a function of retinal position of the target separately for mask inside (central to the target) and mask outside (peripheral to the target) conditions. 
or order of readout to the asymmetry effect. Furthermore, the use of a detection technique makes it unnecessary to isolate the asymmetry effect by taking a mean over conditions in which order of readout was varied. Finally, since the mask was always the same letter and was never a member of the target set, it is unlikely that it caused masking by competing for processing capacity or limited space in shortterm memory. The decline in masking as target-noise distance increased supports this last conclusion, since effects of memory or of order of identification of elements should not vary with the small variation in proximity used in this experiment. It seems clear that lateral masking affects the quality of the sensory information received by the visual system and not the mechanisms of perceptual processing that operate on this information. The only aspect of the experiment that might reasonably lead to a hypothesis of limited processing capacity is the brief exposure duration, and this aspect is eliminated in Experiment 2 .

The present interpretation of the asymmetry of lateral masking ascribes it to the retinal acuity gradient; that is, retinal acuity decreases as the distance from the fovea increases, making recognition of the target more difficult. Masking is more pronounced when the mask is on the peripheral than when it is on the central side of the target, because the target-plus-mask configuration is further removed from the fixation point in the former case than in the latter case. The effective retinal locus of the stimulus should be computed from the midpoint of this configuration, not from the midpoint of the target itself. A target paired with an inside mask should be more easily recognized than a target with an outside mask, because the midpoint of the former pair is in a region of greater retinal acuity than that of a latter. Thus, the acuity gradient curves for the inside and outside mask conditions (as in Figure 3) should be parallel but separated by the number of degrees by which the target-noise pair differ in the two conditions.

Two predictions follow from these considerations. First the retinal acuity gradients for the mask-inside and mask-outside conditions should be parallel. As is seen in Figure 3, this prediction holds up fairly well overall. Separate plots of the acuity gradients for each of the four target-mask separations show equally good parallelism, and the analysis of variance does not support nonparallelism of the functions. The chief departure from parallelism is found in the region of lower acuity, where an increased probability of guessing might be expected to introduce irregularity into the function.

The second prediction is that the parallel acuity gradients should be separated on the $\mathrm{x}$-axis (Figure 3 ) by the mean difference between the retinal locations of the target-noise configuration in the inside and outside conditions. Over the entire experiment, the mean target-noise separation was approximately $18^{\prime}$ and the mean width of letters, $8^{\prime}$. The predicted separation of the functions should therefore be $26^{\prime}$. To visualize the derivation of the $26^{\prime}$, consider Figure 1. To "line-up" the two target-mask pairs so that they would look like those in Figure 2, one would need to shift one or the other by the amount of target-noise separation, plus the width of one letter.

As can be seen in Figure 3, a horizontal shift of $26^{\prime}$ is slightly more than what is necessary to "correct" the two gradients and make them coincide. A least squares estimate of the optimal amount of shift needed is $15.0^{\prime}$. The estimate was obtained by putting the best fitting parallel lines through the two functions and then determining the amount of horizontal shift that would be required to make them coincide. (For mask peripheral, $\mathrm{r}^{2}$ was .967 , for mask central, .929.) By the same reasoning, the predicted shifts in acuity gradients for the four different conditions of target-noise proximity are $18^{\prime}, 23^{\prime}, 28^{\prime}$, and $33^{\prime}$, and corresponding least-square estimates of the optimal amount of shift are 14.3', 8.03', 22.2', and $9.28^{\prime}$, respectively. Although the acuity gradients for the different conditions of target-mask distance have roughly the appearance of Figure 3, they are considerably more variable (having only $25 \%$ of the data apiece), and it is not surprising that the least squares esimates vary as they do. (The $\mathrm{r}^{2} \mathrm{~s}$ for these fits ranged from .80 to .95 , with a mean of .897.)

The results of Experiment 1 generally support the acuity gradient hypothesis of the asymmetry of masking. The gradients for inside and outside masks are approximately parallel, as predicted, and the hypothesis predicts fairly well the amount of shift needed to make the parallel functions coincide. The prediction of the shift is not perfect, but it more than compensates for the asymmetry effect. There is certainly no evidence for an asymmetry effect over and above the acuity gradient that needs to be accounted for by some additional mechanism.

\section{EXPERIMENT 2}

In this experiment, the target letter was stationary at some position in the field and the masking letter was moved until the subject could report the identity of the stationary letter. The positions chosen for the target were ones in which it could be seen eventually. Thus, the subject needed only to wait until the target was visible and should rarely have had to guess the identity of the target. Consequently, the guessing that took place in Experiment 1, and that probably distorted the functions, should not affect the func- 
tions obtained in this experiment. In addition, since this experiment obtains lateral masking functions with unlimited viewing, as in Townsend et al. (1971), the experiment also minimizes the role of memory factors and strategies of readout in the estimate of the amount and of the asymmetry of lateral masking.

\section{Method}

Subjects directed their regard to a fixation point on either the left or right side of a large luminous panel. A single black letter was presented on the panel and it was flanked by a single masking letter, $\mathrm{H}$, which could be either on the inside of the letter (i.e., between the letter and the fixation point) or on the outside of the letter (on the peripheral side of it). One second after the letter and mask were presented, the mask was moved away from the target at a rate of $1 \% \mathrm{sec}$, and the subject, keeping his gaze on the fixation point, attempted to identify the target letter while the mask was being moved. The mask was stopped as soon as the subject correctly identified the target, and the target-mask distance was recorded.

There were 13 target letters: A, G, J, K, L, M, O, S, T, U, V, $\mathrm{X}$, and $\mathrm{Y}$. These and the masking $\mathrm{H}$ were black Rotex rub-on letters (48 Pt/R-1948-C) measuring approximately $20^{\prime}$ wide and $50^{\prime}$ high. The letters were mounted on thin strips of transparent plastic exactly the width of each letter.

The letters were viewed against a large $(56 \mathrm{~cm}$ wide $\times 25 \mathrm{~cm}$ high) back-illuminated milk Plexiglas panel from a distance of $1.9 \mathrm{~m}$. The luminance of the panel was $12 \mathrm{fL}$, and the letters were opaque. The luminous panel was set in a larger black frame and covered by an opaque shutter that was hinged above the panel. The subject fixated a small $\left(10^{\prime}\right)$, bright (approximately 15 to $20 \mathrm{fL}$ ) light on one side of the panel prior to a trial. Once fixation was achieved, the experimenter pulled a cable to raise the shutter, exposing the panel and the stimuli used for that trial. While the subject kept his gaze on the fixation light, the experimenter moved the mask away from the target.

Each subject (two females and one male aged 18-20 years; they all had performed in Experiment 1) had three 1-h sessions on separate days. The first was devoted entirely to practice. During this session, the subjects practiced maintaining a constant fixation and generally became accustomed to the task, the appearance of the letters, and so forth. On each experimental session, all 13 target letters were presented twice at each of three retinal locations $\left(5^{\circ}, 6^{\circ}\right.$, and $7^{\circ}$ from the fixation point). Once when each target was presented the mask was inside the target (between target and fixation point) and once it was outside of it. Each session thus consisted of $78(13 \times 3 \times 2)$ trials, which were presented in a different random order for each subject.

\section{Results}

The threshold of seeing the target was computed for each subject as the median of the mask separations required for seeing it. The observations collected for each of the 13 targets and for left and right visual fields for the 2 days were collapsed, and thresholds were computed for inside and outside mask positions for each of the three retinal locations.

These thresholds are seen in Figure 4, where thresholds for inside and outside mask positions are separately plotted as a function of retinal position. Two effects are apparent in this figure. First, the amount the mask must be moved for the target to be seen increases as the target is placed more periph-

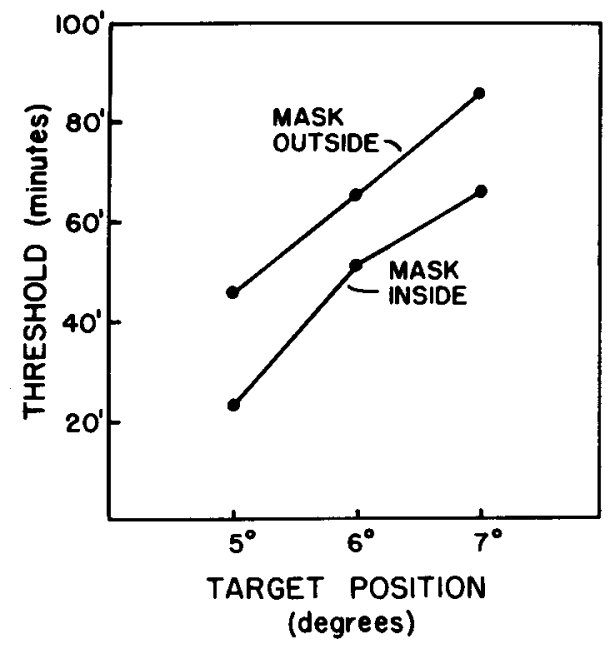

Figure 4. Threshold for seeing the target in Experiment 2 plotted as a function of retinal position of the target separately for mask inside (central to target) and mask outside (peripheral to target) conditions.

erally. This effect is analogous to the decline in acuity as a target is moved away from the fixation point, and it is reliable, with $F(2,4)=27.8, p<.01$. Second, a strong asymmetry of interference is observed. For the target to be seen, the mask must be moved further from the target when it is on the peripheral side of the target than when it is on the foveal side. This effect (asymmetry) is also significant, with an $F(1,2)=26.0, p<.05$. The thresholds for inside and outside placement of the mask trace out statistically parallel functions of retinal position, with an $F=.23$.

The prediction of the acuity gradient hypothesis for the asymmetry of masking in this experiment is most easily shown graphically. The hypothesis, to repeat, states that the crucial variable in detection is the distance of the target-plus-mask cluster, as a whole, from the fixation point. The threshold functions for central and peripheral mask conditions in Figure 4 are plotted according to the retinal position of the target, not the target-mask configuration. According to the hypothesis, the two functions would coincide if the center of the configuration, rather than the target, were used as the point from which the distance to the fixation point was measured.

Figure 5 plots the data of Figure 4 with the correction required by the acuity gradient hypothesis. The correction, applied to both mask peripheral and mask central conditions, consists of half the threshold distance plus half the width of one letter $\left(10^{\prime}\right)$. This correction results from the fact that the center of the configuration is halfway between the target and mask, and the threshold measure gives the full targetmask distance. The additional $10^{\prime}$ is necessary be- 


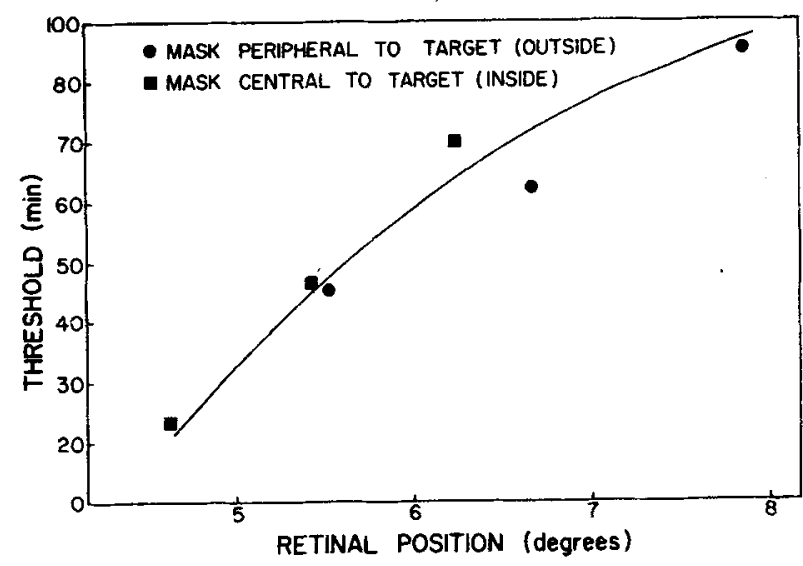

Figure 5. Threshold from Experiment 2 plotted as a function of the retinal position of the midpoint of the target-plus-mask configurations for both mask central and mask peripheral conditions.

cause the target-mask threshold is measured from edge to edge of the letters, and the correction requires a center-to-center distance. The correction is added to the abscissa value for the mask peripheral condition and subtracted from the abscissa value for the mask central conditions.

Figure 5 supports the hypothesis that asymmetry of masking is a result of the retinal acuity gradient. When the peripheral and central masking functions are plotted, as in Figure 5, with the center of gravity as the abscissa, there is no asymmetry effect. The plot shows a common retinal acuity gradient on which stimuli with masks at the peripheral and central ends both fall, at least for the range from approximately $4^{\circ}$ to $8^{\circ}$, in the periphery.

\section{EXPERIMENT 3}

This experiment partially replicates Experiment 2 with monitoring of eye movements. Experiment 2 did not have a procedure for determining whether subjects actually followed instructions to keep their eyes fixated on the assigned fixation points. The subjects could have made unconscious or surreptitious eye movements, despite the instructions and the 1-h training session. A few eye movements occurring at random would have little effect on the data, merely reducing the overall amount of masking found in every condition. However, it is possible that eye movements varied differentially with conditions and thus systematically distorted the masking functions. This experiment was performed to eliminate any effect of eye movements on the functions. A monitoring device was used both to help train subjects to keep their fixation on the correct point and to signal when eye movements occurred during data collection trials. Data were not recorded on trials in which the subject did not maintain fixation.

\section{Method}

The apparatus and letters of Experiment 2 were used. The procedure of Experiment 2 was followed with the following exceptions. First, eye movements were monitored, as described below. Second, training and data collection trials were conducted in a single session approximately $2 \frac{1 / 2}{h}$ long. Third, the task had only 3 possible target letters $(A, V$, and $X)$ rather than the 13 used in Experiment 2. A counterbalancing procedure similar to Experiment 2's was used. Two observations were taken for each target in each position in both visual fields for both central and peripheral masks.

On the day prior to the experimental session, each subject molded a form-fitting athletic mouth protector to his or her upper teeth. This was affixed rigidly to a bite board for each subject, and the bite board was adjusted for each subject to place his or her eyes at the same distance from the screen that was used in Experiment 2. Subjects could rest comfortably with their upper teeth on the bite board, and they could not move their heads when in position on the bite board.

Eye movements were monitored by the scleral reflection technique. An infrared light-emitting diode illuminated the eye (its radiation could not be seen by any of the subjects), and two infrared detectors, each sensing the reflection from a different side of the cornea in the horizontal plane, were connected to the complementary inputs of a differential amplifier. Motions of the eye vary the relative proportion of corneal and scleral reflection picked up by the two infrared detectors and thus vary their relative outputs to the differential amplifier. The output of the differential amplifier was amplified and observed on an oscilloscope. The system could determine the absolute position of the eyes in the horizontal plane to the nearest $1 / 2^{\circ}$. The experiment required two experimenters, one to monitor the oscilloscope and one to adjust the stimuli.

The first part of a session took from 30 to $60 \mathrm{~min}$ and was devoted to training and to accommodation of each subject to the apparatus. The optimal positions for the infrared detectors were found, and the subjects went through about 20 trials in which they were told when they moved their eyes and were generally given feedback and coaching in maintaining their fixation.

During data-collection trials, the subjects were informed when they moved their eyes and the data from such trials were not recorded. The trial that led to the eye movement was repeated later in the block of trials. The criterion for an eye movement on these trials was approximately $1^{\circ}$. This criterion was used rather than the $1 / 2^{\circ}$ of which the apparatus is capable because small eye movements occurred at the beginning of a trial some time before the target was identified. These initial eye movements apparently did not help the subjects identify the target and were, in any event, impossible for the subjects to eliminate completely. The initial eye movements appeared to have two components: (1) an increase in the amplitude of small high-frequency oscillations of the eye and (2) a smooth, nonsaccadic, involuntary tracking response to the shutter as it was raised. Both types of eye movement were clearly distinguishable from saccadic inspections of the array, both in velocity and in extent of maximum excursion.

There were four subjects, three being students and the fourth, W.B., being the first author. Subjects W.B. and A.L. had $20 / 12$ uncorrected vision, measured with a Snellen eye chart, T.R. had $20 / 20$ corrected vision (he was able to wear his glasses over the eye monitoring apparatus), and W.S. had 20/20 uncorrected vision.

\section{Results}

Very few eye movements were detected during data-collection trials. There were 72 data-collection trials, but only two of these had to be repeated for W.S. because of an eye movement, four each for T.R. and W.B., and 10 for A.L. (The number for A.L. 
is approximate, because the eye monitoring device fit her poorly and sometimes gave spurious readings.) It is tempting to conclude that few eye movements occurred in Experiment 2, but training in Experiment 2 did not include eye monitoring and thus may not have been as successful.

Figure 6 plots the mean target-mask separation (measured from the center of the target to the center of the mask) required for identification of the target. The abscissa gives the retinal position of the target, and data are plotted separately for mask central and mask peripheral conditions. Twelve observations were taken for each point for each subject, but observations that were more than \pm 2 standard deviations away from the mean were omitted from the means plotted in Figure 6. As it turned out, all of the observations dropped were more than +2 standard deviations; none was dropped because it was too small. Two observations were dropped from T.R.'s data (both from mask central conditions, one at $6^{\circ}$ and one at $7^{\circ}$ target location), two from W.B.'s data (both from mask peripheral conditions, one from the $5^{\circ}$ and one from the $6^{\circ}$ target location), and four from A.L.'s data (one each from $6^{\circ}$ and $7^{\circ}$ for mask peripheral and $5^{\circ}$ and $6^{\circ}$ for mask central conditions). None of W.S.'s data was dropped.

Figure 6 shows a strong effect of asymmetry of masking, as well as a consistent retinal acuity gradient. The asymmetry effect seems quite reliable; in only one case (A.L. at $5^{\circ}$ ) are the corresponding mask central and mask peripheral points within 2 standard errors of each other, and in most cases they are separated by much more than that. The absolute amount of lateral masking for T.R., W.B., and A.L. is about the same as was shown by the subjects in Experiment 2, but W.S. had quite a bit more, his thresholds being about twice those of the other subjects. The differences between the results of Experiments 2 and 3 do not show any systematic trends that might be attributable to differences in the number of eye movements in the two cases.

Figure 7 shows the thresholds of Figure 6 plotted with the center of the line connecting the midpoints of the target and noise as the abscissa. Figure 7 bears the same relation to Figure 6 as Figure 5 does to Figure 4; in both Figures 5 and 7, the masking functions are "corrected" so that masking is plotted as a function of the retinal position of the target-mask pair as a whole rather than of just the target. It seems clear from Figure 7 that the correction procedure is fairly successful. It compensates almost perfectly for the asymmetry effect for W.S. and T.R., and it very nearly removes all of the asymmetry effect for W.B. and A.L. It appears, as was the case in Experiment 2, that the corrected functions trace a single acuity gradient on which thresholds arising from mask central and mask peripheral conditions both fall. One measure of the goodness of fit of a single acuity gradient is the linear regression of retinal position on threshold. Linear fits are drawn in Figure 7 , and the $\mathrm{r}^{2}$ for the regression is $.93, .97, .86$,

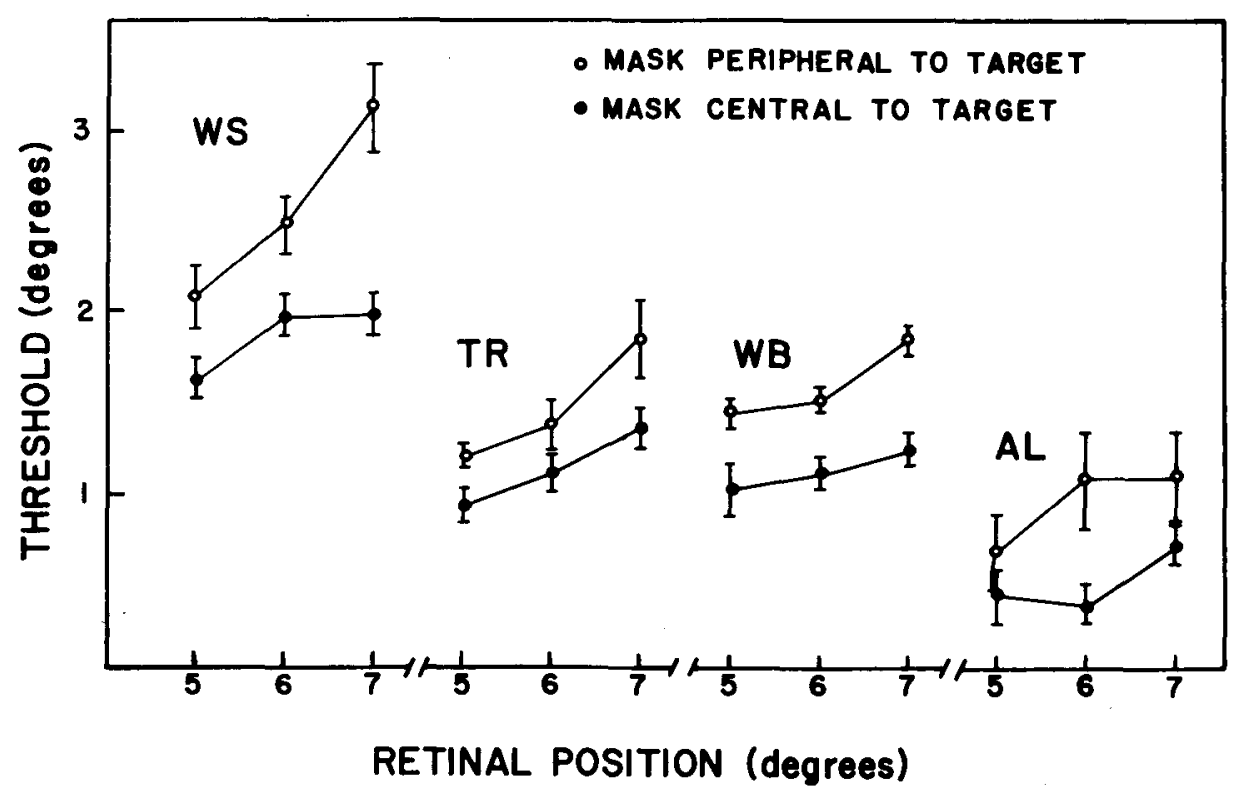

Figure 6. Threshold target-mask difference for seeing the target in Experiment 3 plotted as a function of retinal position of the target separately for mask central (closed circles) and mark peripheral (open circles) conditions. The plots are presented separately for each of the four subjects, identified by initials. The \pm standard error range about each mean is indicated by the vertical bars through each point. 


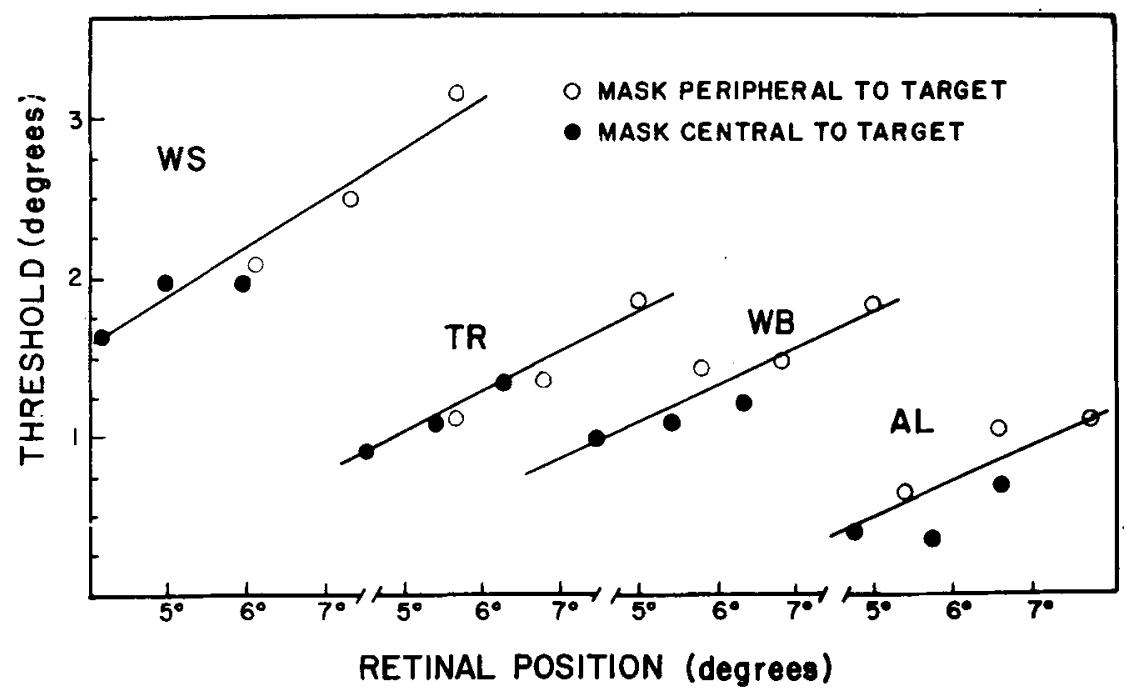

Figure 7. Thresholds from Experiment 3 (Figure 6) plotted as a function of the retinal position of the target-plus-mask cluster for both mask central and mask peripheral conditions.

and .67 for W.S., T.R., W.B., and A.L., respectively. An indication of the degree to which the correction of works is given by the $r^{2} s$ for the regression prior to the correction. These are .35 , $.63, .22$, and .27 for W.S., T.R., W.B., and A.L.

\section{DISCUSSION}

The results of these experiments seem to leave little doubt that lateral masking among elements in the visual field results at least in part from sensory interference. In Experiment 1, the use of a forced-choice detection technique rather than "whole report," the limitation of the size of the array to just two items, and the use of a constant masking element which is never a target all serve to eliminate explanations of the results based on memory limitations or strategies of inspection of the array. The second and third experiments show, in addition, that lateral masking still takes place with unlimited viewing of stimuli constructed with the constraints of those in Experiment 1.

The experiments also support the retinal acuity gradient as a major source of asymmetry of visual masking. When the target is kept at a constant position in the field as the mask is moved about, the target-plus-mask configuration is, as a whole, more peripherally located in the field with a mask peripheral to the target than with a mask central to the target. If some component of lateral interference comes from confusion of features in the target and mask letters, then the retinal acuity gradient alone would predict more masking with a peripheral mask than with a central one. In these experiments, when the overall position of the mask-target cluster in the field is equated for the peripheral and central mask conditions, the asymmetry of masking is eliminated. The equating procedure actually overcorrects for, and thus reverses, the asymmetry effect in the first experiment. This may be because the presence of guesses in the data reduced the measured asymmetry effect. It could also be because the correction procedure used a linear regression and the acuity gradient in Experiment 1 is nonlinear. In Experiment 2, subjects guessed very infrequently (error rate was $3 \%$ ) and the correction procedure did not rely on a regression technique. Possibly because of these factors, the correction compensates for the asymmetry effect almost exactly in Experiment 2 . In Experiment 3 , the correction perfectly compensates for asymmetry of masking for two subjects and slightly undercompensates for two. It is possible that some asymmetry of masking still exists after the retinal acuity gradient is accounted for, but the asymmetry is not very strong, at least when nonsensory factors are controlled.

Wolford and Hollingsworth (1974) have suggested something similar to the retinal acuity hypothesis to account for the asymmetry of lateral masking. They proposed that contour interaction among letters increases as they are placed nearer the periphery. Thus, replacing a peripheral masking letter with a blank space would aid recognition of a target more than replacing a central masking letter with a blank space, and an asymmetry effect would be observed when central and peripheral masking conditions are compared. The contour interaction hypothesis is actually a special case of the retinal acuity 
gradient hypothesis, since contour interaction is just one factor that could contribute to the centralperipheral gradient for detecting letters. Refractive error, larger receptive fields, or a lower density of receptors in the periphery could also be factors in the acuity gradient even if there were no contour interactions at all.

It is true that the central-peripheral acuity gradient is much steeper when there are a number of adjacent letters in the field than when there is only an isolated target letter (Estes et al., 1976; Mackworth, 1965). However, the steeper acuity gradient found with multiple letters does not require that a dynamic process of lateral masking or contour interference take place among adjacent letters. The fact is simply that the acuity gradient for multiple letters cannot be predicted from the gradient for a single letter. The multiple letters could give a steeper gradient because of acuity factors that do not exist with a single letter. With two or more letters in the field, the blank space between letters is itself a feature whose detection is essential for recognition of the letters. Detection of the blank space could decline as the letters move toward the periphery and cause recognition accuracy for any one of the letters to decline more rapidly than it would if the letter were alone in the field. Of course, these arguments do not deny that a process of inhibitory contour interaction among letters takes place. They are only intended to support other factors that might lead to a steeper central-peripheral acuity gradient for multiple than for single letters.

Estes et al. (1976) have proposed that at least some portion of lateral interference between letters in the periphery comes from uncertainty about the retinal position at which features are located, and they propose, further, that this positional uncertainty increases with distance in the periphery. Their proposal of positional uncertainty is consistent with the acuity gradient interpretation of the asymmetry of masking. However, Estes et al. also propose that the gradient of positional uncertainty is skewed such that the location of a feature will be taken as being more central than it actually is in the field. Such a skewed positional uncertainty gradient would certainly create an asymmetrical masking function, but the question is whether it is necessary to hypothesize a skewed gradient. The present asymmetry effects do not need any explanation over and above the retinal acuity gradient. In the Estes et al. data, on the other hand, the asymmetry effect appears to be somewhat larger than the acuity gradient can account for, and a factor operating in addition to the retinal acuity gradient would therefore seem necessary to account for their data.

Although the Estes et al. experiment differs in a number of ways from the present ones, the critical difference that necessitates an extra factor to explain their asymmetry effect may be the fact that their masking letters were also targets that had to be identified at the same time as the letters they masked. Thus, it was more difficult for the subjects of Estes et al. to distinguish the features that intruded on the target letter from the mask than it was for our subjects. Our subjects knew the masking letter was always an $\mathbf{H}$, and they were presumably quite practiced at distinguishing its features from those of other letters. If this interpretation of the difference between the experiments is correct, then the extra amount of asymmetry effect in the Estes et al. experiment comes from a stage of processing after the intake of featural information. It is not a result of interference with the intake of sensory information. Our experiments, in which an increase in lateral masking is not accompanied by an increase in the difficulty of the decision about the letter implied by the featural information, presumably measure lateral masking at the sensory level, while the Estes et al. results contain both sensory masking and interference at an early stage of perceptual decision making. We conclude, then, that the asymmetry of masking at the sensory level is almost entirely accounted for by the retinal acuity gradient.

\section{REFERENCES}

Eriksen, B. A., \& Eriksen, C. W. Effects of noise letters upon the identification of a target letter in a nonsearch task. Perception \& Psychophysics, 1974, 16, 143-149.

Estes, W. K., Allmeyer, D. H., \& Reder, S. M. Serial position functions for letter identification at brief and extended exposure durations. Perception \& Psychophysics, 1976, 19, 1-15.

Estes, W. K., \& WoLford, G. L. Effects of spaces on report from tachistoscopically presented letter strings. Psychonomic Science, 1971, 25, 77.80.

Korte, W. Uber die Gestaltauffassung im indirecten Sehen. Zeitschrift für Psychologie, 1923, 93, 17-82.

Mackworth, N. H. Visual noise causes tunnel vision. Psychonomic Science, 1965, 3, 67-68.

SHAw, P. Processing of tachistoscopic displays with controlled order of characters and spaces. Perception \& Psychophysics, $1969,6,257-266$.

TAYloR, S. G., \& BRown, D. R. Lateral visual masking: Supraretinal effects when viewing linear arrays with unlimited viewing time. Perception \& Psychophysics, 1972, 12, 97-99.

Townsend, J. T., TAYlor, S. G., \& Brown, D. R. Lateral masking for letters with unlimited viewing time. Perception \& Psychophysics, 1971, 10, 375-378.

Wolford, G. L., \& Hollingsworth, S. Retinal location and string position as important variables in visual information processing. Perception \& Psychophysics, 1974, 16, 437.442.

Wolford, G. L. Perturbation model for letter identification. Psychological Review, 1975, 82, 184-199.

WOODWORTH, R. S. Experimental psychology. New York: Holt. 1938.

(Received for publication December 27, 1976; revision accepted June 28,1977 .) 